\title{
On the oxidation mechanism of C1-C2 organic molecules on platinum.
}

\section{A comparative analysis.}

\author{
Rubén Rizo, Rosa M. Arán-Ais, Enrique Herrero* \\ Instituto de Electroquímica, Universidad de Alicante, \\ Apdo. 99, E-03080, Alicante, Spain. \\ e-mail:herrero@ua.es
}

\begin{abstract}
The rationale design of better electrocatalysts for the oxidation of $\mathrm{C} 1-\mathrm{C} 2$ organic molecules requires the detailed knowledge of their oxidation mechanism. Pt single crystals are powerful tools to study, in a simple way, the surface structure effect on the different reaction pathways. Here, the oxidation mechanism of these molecules is compared so that the knowledge gained with the simpler mechanism is transferred in the analysis of the more complex ones. The goal is to design strategies so that the platinum electrodes improve their performance in their oxidation by the appropriate modification with other elements by attacking the bottlenecks in the reaction mechanism.
\end{abstract}




\section{Highlights}

- Similarities and differences between the oxidation mechanisms of C1-C2 molecules are highlighted.

- The active pathway of formic acid oxidation through $\mathrm{CO}_{2}$ requires the adsorption of monodentate formate.

- A surface covered by adsorbed $\mathrm{OH}$ is required for methanol and ethanol oxidation reactions.

- Greater reactivity towards the $\mathrm{C}-\mathrm{C}$ cleavage by partially oxidizing the $\alpha$ carbon of ethanol molecule. 


\section{Introduction.}

Small organic molecules, especially those $\mathrm{C} 1$ and $\mathrm{C} 2$, have been proposed to be used as combustible in fuel cells to generate electricity from the chemical energy stored in their bonds. Those molecules present the advantage with respect to hydrogen that can be obtained from renewable sources, i.e., biomass and they are liquids that can be distributed using the present network used for the oil products. However, the overpotentials for the oxidation reaction of these molecules on the available electrodes is significantly higher than that measured for the hydrogen oxidation reaction, which diminishes the efficiency of these devices. For this reason, additional research is required in the quest of more efficient electrocatalysts. To reach this goal, a detailed mechanism for the oxidation reaction is required, so that the elemental steps are identified, and the key factors governing the electrocatalysis are known. The oxidation mechanism of these molecules shares many common elements, and the knowledge acquired in one can be transferred to the other and better practical electrocatalysts can be prepared in rational way.

From all the $\mathrm{C} 1-\mathrm{C} 2$ molecules, formic acid is the one that presents the simplest mechanism in the oxidation to $\mathrm{CO}_{2}$. This process involves the exchange of 2 electron and 2 protons. From a chemical point of view, the oxidation reaction requires the cleavage of a $\mathrm{C}-\mathrm{H}$ and an $\mathrm{O}-\mathrm{H}$ bond. $\mathrm{Pt}$ and $\mathrm{Pd}$ electrodes have been shown to have a good performance for the formic acid oxidation reaction (FAOR) [1,2]. The main characteristic of these metals is their affinity for hydrogen, which is readily adsorbed on these surfaces.

The next molecule in the series is methanol. In this case, the complete oxidation (which involves the exchange of 6 electrons) requires not only the cleavage of several bonds, but also the formation of new ones. Thus, three $\mathrm{C}-\mathrm{H}$ bonds have to be cleaved 
and also an $\mathrm{O}-\mathrm{H}$ bond in the alcoholic group. On the other hand, a new $\mathrm{C}-\mathrm{O}$ bond has to be formed so that $\mathrm{CO}_{2}$ is obtained. The increasing complexity of this reaction is translated to the practical results [3], and thus oxidation currents are smaller and the overpotentials are higher than those measured for formic acid.

The equivalents for formic acid and methanol in $\mathrm{C} 2$ molecules are acetic acid and ethanol. The complete oxidation to $\mathrm{CO}_{2}$, in which 8 and 12 electrons are transferred, respectively, involves an additional step not considered in the previous oxidation mechanisms, the $\mathrm{C}-\mathrm{C}$ bond cleavage, which appears to be the most difficult step [4-6]. In fact, acetic acid is stable on platinum at room temperature and it is only oxidized at very high potential [7]. On the other hand, when the oxidation of ethanol is considered, a mixture of acetaldehyde, acetic acid and $\mathrm{CO}_{2}$ is obtained, depending on the operating conditions and the electrode properties. The formation of species different from $\mathrm{CO}_{2}$ implies the diminution in the efficiency of the reaction, since the formation of acetaldehyde or acetic acid involves the transfer of 2 and 4 electrons, respectively.

All these $\mathrm{C} 1-\mathrm{C} 2$ molecules share common characteristics and therefore the knowledge acquired when studying one of these molecules can be applied to the other molecules. One of the shared features of all the mechanism is the existence of several pathways in the reaction mechanism, which make difficult to identify the key elements in it. In fact, the FAOR, which has the simplest reaction mechanism, already have a dual pathway: the direct path involves an active intermediate whereas the secondary path implies the formation of adsorbed $\mathrm{CO}$ as intermediate species, which act as a poison [8]. Despite all these common features, the translation of the knowledge from one to the other is not straightforward and has to be adapted to the specific properties of the studied molecule. In this review, the similarities, and differences between the oxidation of these molecules will be highlighted so that a better understanding of the oxidation 
mechanisms can be achieved, with the final aim of synthesizing tailored anode electrocatalysts for fuel cells.

\section{The formic acid oxidation reaction (FAOR).}

In principle, any oxidation mechanism that involves the exchange of two electrons, such as that of formic acid, should be simple. However, as will be shown, several complications appear in the formic acid oxidation mechanism. As aforementioned, the oxidation requires the cleavage of two bonds. The first one, the $\mathrm{O}-\mathrm{H}$ bond breaking is a trivial step because it is involved in the acid base equilibria of the molecule. In fact, formate has been identified as the active species in the reaction [9-12], and thus, the only relevant bond that has to be cleaved is the $\mathrm{C}-\mathrm{H}$ bond. The knowledge gained in the last years about this reaction has increased significantly using the combination of experimental and theoretical results and has been able to establish the rate determining step in the reaction. These results have been reviewed in previous articles $[2,13,14]$ and here, the most important features will be summarized. First, it is clear that the effective cleavage of the $\mathrm{C}-\mathrm{H}$ bond requires the adsorption of the molecule in the right configuration, that in which the $\mathrm{H}$ atom can interact with the surface. However, the most stable adsorbed configuration of formate, the bidentate form (figure 1A), has been shown to be inactive for the oxidation $[11,12,15]$ because the $\mathrm{H}$ atom is far from the surface and thus, the kinetic barrier for the cleavage of the $\mathrm{C}-\mathrm{H}$ bond is very high (Figure 1A) [2,16]. 
A) Monodentate formate Bidentate formate (favorable for oxidation) (unfavorable for oxidation)
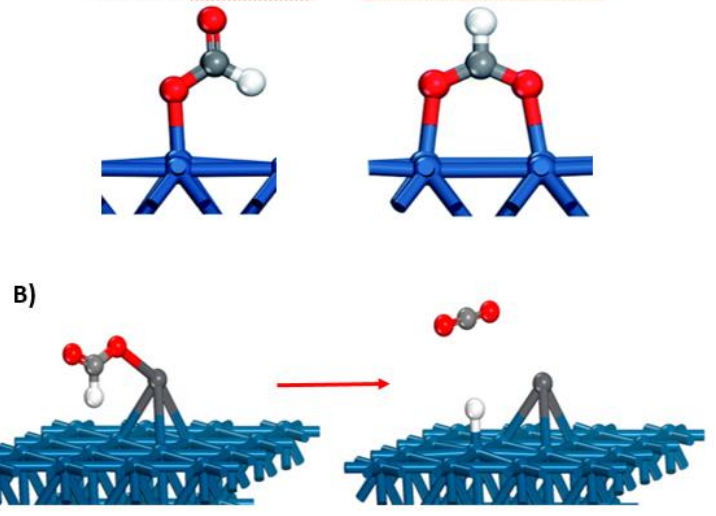

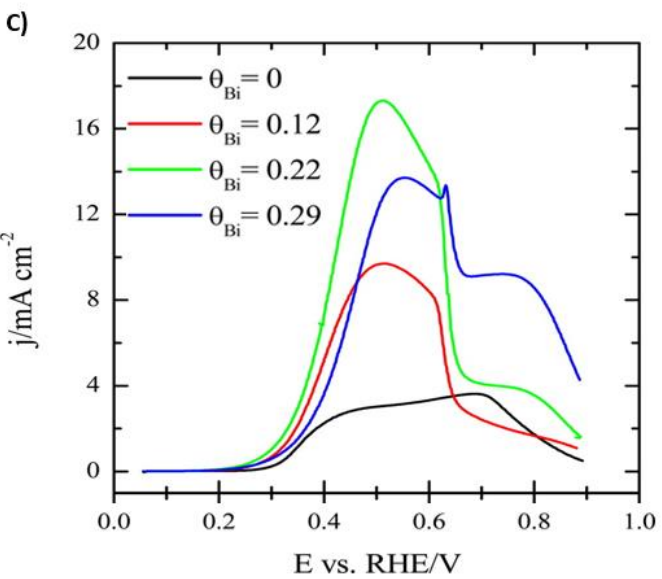

Figure 1. A) Monodentate and bidentate adsorbed formate on a platinum single crystal. Adapted from ref. [2]. B) Geometries of the HCOO fragment chemisorbed on the adatom- $\mathrm{Pt}(111)$ surface and the final products yielded from the formic acid oxidation. Adapted from ref [17]. C) Voltammetric profiles for the $\mathrm{Bi}-\mathrm{Pt}(111)$ electrode for different bismuth coverages in a $0.5 \mathrm{M} \mathrm{H}_{2} \mathrm{SO}_{4}+0.1 \mathrm{M} \mathrm{HCOOH}$ solution at $25{ }^{\circ} \mathrm{C}$. Scan rate: $50 \mathrm{mV} \mathrm{s}^{-1}$. Adapted from ref [17].

The activation of the $\mathrm{C}-\mathrm{H}$ bond, then requires that formate is adsorbed in a monodentate configuration (Figure 1A) [11]. In this position, the $\mathrm{C}-\mathrm{H}$ bond can point to the surface so that the interaction of the hydrogen atom with the surface cleaves the bond in a barrierless step, as DFT studies demonstrate $[11,16]$. To maintain the formate adsorbed in this configuration and preventing the evolution of this adsorption mode to the most stable one, the bidentate formate [2,11], additional adsorbed anions [18-20] or adatoms of the p-block are required [17,21,22] because they prevent the evolution of the monodentate form into the most stable bidentate adsorbed form. If this monodentate form is favored, the reaction rate is very fast and this reaction path is going directly to $\mathrm{CO}_{2}$ formation, leading to high formic acid oxidation currents (Figure 1B-C) [17]. In fact, under these conditions, the rate is limited by the availability of adsorption sites because is independent of the formate concentration [12].

However, the reaction has a second path in which adsorbed $\mathrm{CO}$ is formed and then oxidized at high potentials [23]. In this case, the formation of $\mathrm{CO}$ is an unwanted 
outcome, because it acts as a catalytic poison. The analysis of the kinetics of both paths, the direct one and that going through $\mathrm{CO}$ indicates that the rate of the $\mathrm{CO}$ path is at least two orders of magnitude lower than that of the main path [11]. Despite this much lower reaction rate, the accumulation of adsorbed $\mathrm{CO}$ on the surface eventually controls the reaction rate because it blocks the surface and prevents the adsorption of formate in the monodentate configuration. This effect highlights the importance of side paths in the overall performance of the reaction.

\section{Methanol oxidation reaction (MOR).}

Following the same scheme as that used for the FAOR, the first step would be to determine whether there is a strong interaction of methanol with the surface, because this interaction is required for an effective catalysis. For formate, the detection of adsorption modes was possible using Fourier-transform infrared spectroscopy (FTIR) in the total attenuated reflection (ATR) mode $[10,24,25]$ or voltammetry at high scan rates $[12,26]$. In this case, since methanol oxidation currents are significantly smaller, the voltammetric profiles obtained in solutions with low methanol concentration can be used for that. In perchloric acid solutions, the results indicate that methanol does not adsorb on the surface, at least in the low potential region (figure $2 \mathrm{~A}$ ). In fact, the hydrogen adsorption profile in this region is exactly the same as that obtained in its absence during the first scan for platinum single crystals [27]. 
A)

E vs. RHE/V
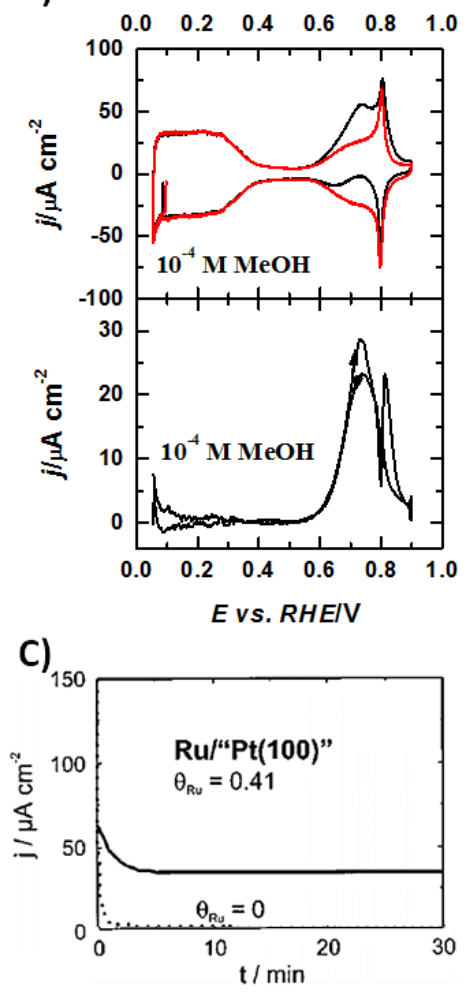

B)
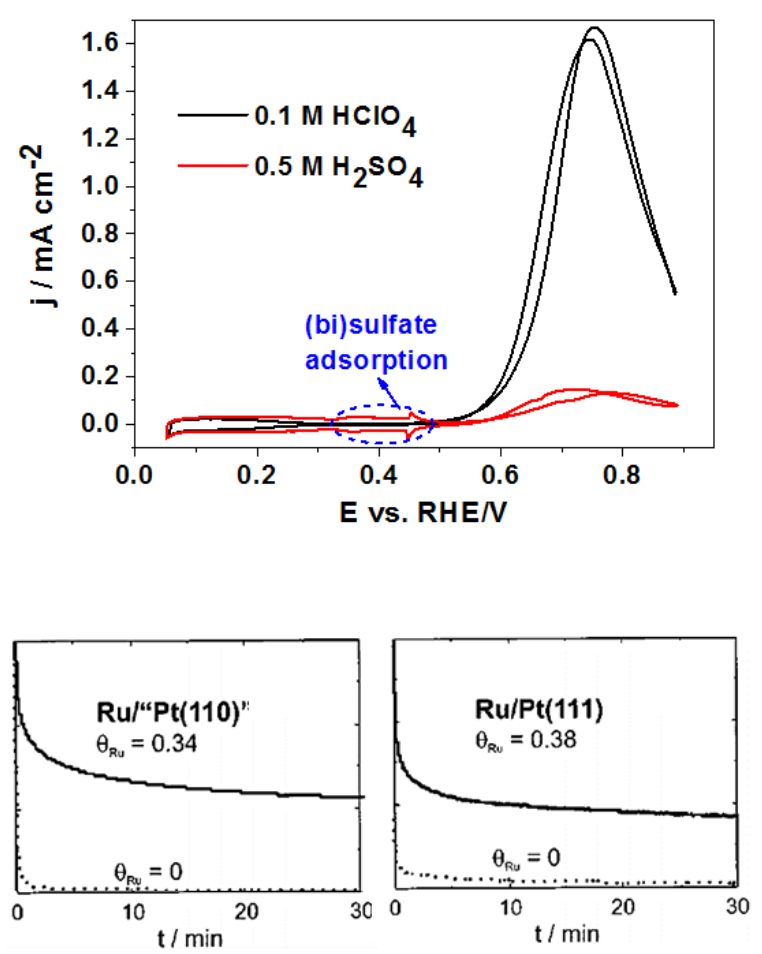

Figure 2. A) Voltammetric profiles of the $\mathrm{Pt}(111)$ electrode in $0.1 \mathrm{M} \mathrm{HClO}_{4}+10^{-4} \mathrm{M}$ $\mathrm{MeOH}$. The red trace shows the profile in absence of $\mathrm{MeOH}$. Subtraction of the profiles in bottom panel. Scan rate: $50 \mathrm{mV} \mathrm{s}^{-1}$. Adapted from ref. [27]. B) Voltammetric profiles of the $\mathrm{Pt}(111)$ electrode in $0.5 \mathrm{M} \mathrm{MeOH}+$ the respective electrolyte. Scan rate: $50 \mathrm{mV} \mathrm{s}^{-1}$. C) Chronoamperometric curves recorded at $0.3 \mathrm{~V}$ vs. $\mathrm{Ag} / \mathrm{AgCl}$ for $\mathrm{Pt}(100)$, $\mathrm{Pt}$ (110) and $\mathrm{Pt}(111)$ in $0.1 \mathrm{M} \mathrm{HClO}_{4}+0.6 \mathrm{M} \mathrm{MeOH}$ before and after $\mathrm{Ru}$ deposition. Adapted from ref. [28].

Probably, the best example is the $\operatorname{Pt}(111)$ electrode, which displays very small currents for low methanol concentrations (figure 2A) [27]. Under these conditions, only small currents are observed above $0.6 \mathrm{~V}$ when $\mathrm{OH}$ is adsorbed on the surface, whereas the rest of the voltammetric profile during the first scan coincides with that obtained in the absence of methanol. A similar situation is observed for the $\operatorname{Pt}(100)$ electrode for potentials below $0.4 \mathrm{~V}$ [27]. The absence of changes in the profile in the presence of methanol indicates that the interaction of methanol with the surface is weak. Indeed, when strongly adsorbed anions are present in the electrolyte, such as sulfate or phosphate [29], currents diminish significantly, even for relatively high methanol 
concentrations and the typical profile observed in the absence of methanol is superimposed to the small methanol oxidation currents. Thus, the typical phase transition in the adsorbed sulfate layer on the $\mathrm{Pt}(111)$ electrode can be still observed in the presence of methanol (figure 2B), implying that the presence of methanol has not disturbed the adsorption of sulfate [30-32]. The large decrease in the currents in the presence of specifically adsorbed anions and the observation of the typical features of the supporting electrolyte clearly implies that the interaction with the surface is very weak, which makes the electrocatalysis more difficult.

The second important consequence of the previous results is that the oxidation of methanol requires a surface covered by adsorbed $\mathrm{OH}$. Having this result in mind, and considering the dehydrogenation capabilities of platinum shown with the FAOR, it can be proposed that the oxidation mechanism starts with three dehydrogenation steps to form adsorbed CO. Moreover, it has been shown on a cyanide-Pt(111)-modified electrode that the indirect pathway through adsorbed $\mathrm{CO}$ is a site-demanding process that requires wide atomic arrangements with at least three contiguous atoms of platinum [33]. Then, this adsorbed $\mathrm{CO}$ is oxidized through the well-known LangmuirHinselwood mechanism with the aid of adsorbed $\mathrm{OH}$. This would explain the necessity of adsorbed $\mathrm{OH}$. However, the results indicate that adsorbed $\mathrm{OH}$ not only is required for the oxidation of $\mathrm{CO}$ but also for additional steps. First, the kinetic analysis of transients shows that $\mathrm{CO}$ is only formed on $\operatorname{Pt}(111)$ and $\mathrm{Pt}(100)$ at $\mathrm{E}>0.6 \mathrm{~V}$ and $\mathrm{E}>0.35 \mathrm{~V}$, respectively [31]. Both potentials coincide with the onset of $\mathrm{OH}$ adsorption. Second, the observed kinetic isotopic effects using $\mathrm{CD}_{3} \mathrm{OH}$ imply that the cleavage of a $\mathrm{C}-\mathrm{H}$ bond is involved in the rate determining step [29]. All these results point out the importance of adsorbed $\mathrm{OH}$ on the surface for the dehydrogenation steps, which are a bottleneck in the reaction rate. This will also explain the significant diminution of the oxidation 
currents in the presence of adsorbed anions, such as sulfate or phosphate [29], because these anions hinder the adsorption of $\mathrm{OH}$.

The previous discussion implies a change in the paradigm in the methanol oxidation mechanism. When trying to identify the mechanism by DFT modeling, the model surface for the calculation of the different elemental steps have been always a clean surface in the absence of any adsorbate [34,35]. However, these results point out to the necessity of adsorbed $\mathrm{OH}$ in the platinum surface. Thus, any reliable model should contain some adsorbed $\mathrm{OH}$ species on the interface, so that the interaction of these species with the incoming methanol molecules can be analyzed and considered on the mechanism. In fact, the presence of adsorbed $\mathrm{OH}$ can explain the differences between the behavior of methanol in UHV and water environments [36,37]. Previously, the origin of these differences was assigned to the presence of water that may alter the interaction of the methanol molecules with the platinum surface [37]. However, the main difference is the surface state, whereas a clean surface is used in UHV studies, in electrochemistry, the surface state when methanol oxidation takes place is different, because it is covered by $\mathrm{OH}$. In this sense, the bimetallic combination PtRu has been shown to be a promising anode catalyst material owing its greater performance for MOR and the electrooxidation of CO (Figure 2C) [28]. The improved electrocatalysis has been explained by a bifunctional mechanism, in which the higher affinity of Ru for $\mathrm{OH}$ promotes the oxidation of $\mathrm{CO}[28,38,39]$. From the above results, $\mathrm{Ru}$ atoms will not only help in the oxidation of $\mathrm{CO}$, but also may play an important role in the dehydrogenation steps, since $\mathrm{OH}$ is required. 


\section{Ethanol and ethylenglicol oxidation reactions (EOR and EGOR).}

The EOR at low temperatures have two possible final products. The first one is $\mathrm{CO}_{2}$, which implies that the $\mathrm{C}-\mathrm{C}$ bond has been cleaved. The second one is acetic acid, which maintains the $\mathrm{C}-\mathrm{C}$ bond intact. To obtain the maximum energy from the reaction, that is, the 12 electrons that can be finally exchanged, the cleavage of the $\mathrm{C}-$ $\mathrm{C}$ bond is required. However, this step is the most difficult one, because the main products of the oxidation at room temperature are acetic acid or acetaldehyde (the previous step in the formation of acetic acid).

When studying the reaction in acidic solutions on $\mathrm{Pt}(111)$ electrodes, no $\mathrm{CO}_{2}$ can be detected on defect-free electrodes $[4,40]$, implying that defects are involved in the cleavage of the $\mathrm{C}-\mathrm{C}$ bond. On the other hand, in perchloric acid, the initial currents in the transients for the oxidation of ethanol are very high for $\mathrm{E}>0.6 \mathrm{~V}$ [4], where $\mathrm{OH}$ is adsorbed on the electrode surface. This fact indicates that the oxidation of ethanol to yield acetic acid is very active in this potential region. The reaction to form acetic acid requires the transfer of an oxygen group, which is facilitated by the presence of adsorbed $\mathrm{OH}$ through a Langmuir-Hinselwood mechanism. Thus, ethanol molecules interact with the Pt surface covered by $\mathrm{OH}$ and acetic acid is formed. Although the initial current in the transients is very high, the transients show a fast decay. In fact, the current after 1 second can be ten times smaller than the initial current. The decay is due to the adsorption of acetate from acetic acid, which is detected by FTIR [4]. Acetate is adsorbed in the bidentate configuration, the typical adsorption configuration for the carboxylic groups on metals [41]. Two consequences can be extracted from this behavior: i) $\mathrm{OH}$ is required in the oxidation of ethanol to acetaldehyde/acetic acid, ii) the interaction of ethanol with the surface is weak, because other adsorbed species significantly diminishes the oxidation currents. Moreover, as happens with formic acid, 
the adsorption of acetate in bidentate configuration is inactive for the further oxidation of this species because none of the atoms in the adsorbed species can interact readily with the surface. In alkaline solutions, where acetate is not adsorbed, voltammetric currents are significantly larger and the decay in the currents is much slower, which support these conclusions [42]. In this case, the observed decay is due to the polymerization of acetaldehyde, which is produced also with acetic acid in alkaline solutions, leading to the blockage of the electrode surface.

Regarding the cleavage of the $\mathrm{C}-\mathrm{C}$ bond, the studies indicate that it requires special conditions. FTIR experiments carried out on Pt basal planes demonstrated that the $\mathrm{C}-\mathrm{C}$ cleavage is strongly influenced by the electrode surface structure. Thus, the $\operatorname{Pt}(110)$ electrode displays the highest activity for the $\mathrm{C}-\mathrm{C}$ bond splitting, whereas this step is very unfavorable on $\operatorname{Pt}(111)$ electrodes, producing mostly acetic acid during the reaction [4]. DFT calculations demonstrated that the reason of the poor ability of $\mathrm{Pt}(111)$ to oxidize ethanol to $\mathrm{CO}_{2}$ is related with its higher activation barrier for $\mathrm{C}-\mathrm{C}$ bond breaking $(1.36 \mathrm{eV})$ when compared to $\mathrm{Pt}(100)(0.65 \mathrm{eV})$ and that the bond cleavage occurs through strongly chemisorbed precursors, such as $\mathrm{CH}_{2} \mathrm{CO}$ or $\mathrm{CHCO}$, only at low-coordinated surface sites [43] or (110) steps on (111) terraces [41], corroborating the experimental results [44].

Studies with isotopically labelled ethanol $\left({ }^{12} \mathrm{CH}_{3}{ }^{13} \mathrm{CH}_{2} \mathrm{OH}\right)$ have been also able to produce very valuable information on how this bond cleavage takes place on the step sites [45]. The cleavage takes place at low potentials $(\mathrm{E}<0.2 \mathrm{~V})$ and produce two fragments. The ${ }^{13} \mathrm{CH}_{2} \mathrm{OH}$ fragment evolves immediately to adsorbed ${ }^{13} \mathrm{CO}$, which is eventually oxidized to ${ }^{13} \mathrm{CO}_{2}$ above $0.6 \mathrm{~V}$. On polycrystalline $\mathrm{Pt}$ surfaces, it have stablished that the other fragment, ${ }^{12} \mathrm{CH}_{3}$ reduces to $\mathrm{CH}_{4}$ at low potentials during the 
first positive scan, with the aid of $\mathrm{H}_{\mathrm{ad}}$ on (100) sites, present during the first scan (Figure 3A) [46]:

$$
\mathrm{H}_{\mathrm{ad}}(100)+\mathrm{CHOHCH}_{3, \mathrm{ad}} \rightarrow \mathrm{CO}_{\mathrm{ad}}+\mathrm{CH}_{4}+2 \mathrm{H}^{+}+2 \mathrm{e}^{-}
$$

In parallel to the production of methane, the coverage of adsorbed carbon monoxide increases. The formation of $\mathrm{CH}_{4}$ explains why in the isotopically labelled ethanol experiments the amount of ${ }^{13} \mathrm{CO}_{2}$ is always higher than that of ${ }^{12} \mathrm{CO}_{2}$, during the first scan. Only when the $\mathrm{C}-\mathrm{C}$ bond cleavage takes place above $0.5 \mathrm{~V}$, the ${ }^{12} \mathrm{CH}_{3}$ fragment yields adsorbed ${ }^{12} \mathrm{CO}$. This step requires the presence of adsorbed $\mathrm{OH}$ on the surface, highlight the importance of adsorbed $\mathrm{OH}$ in the oxidation of the molecule.
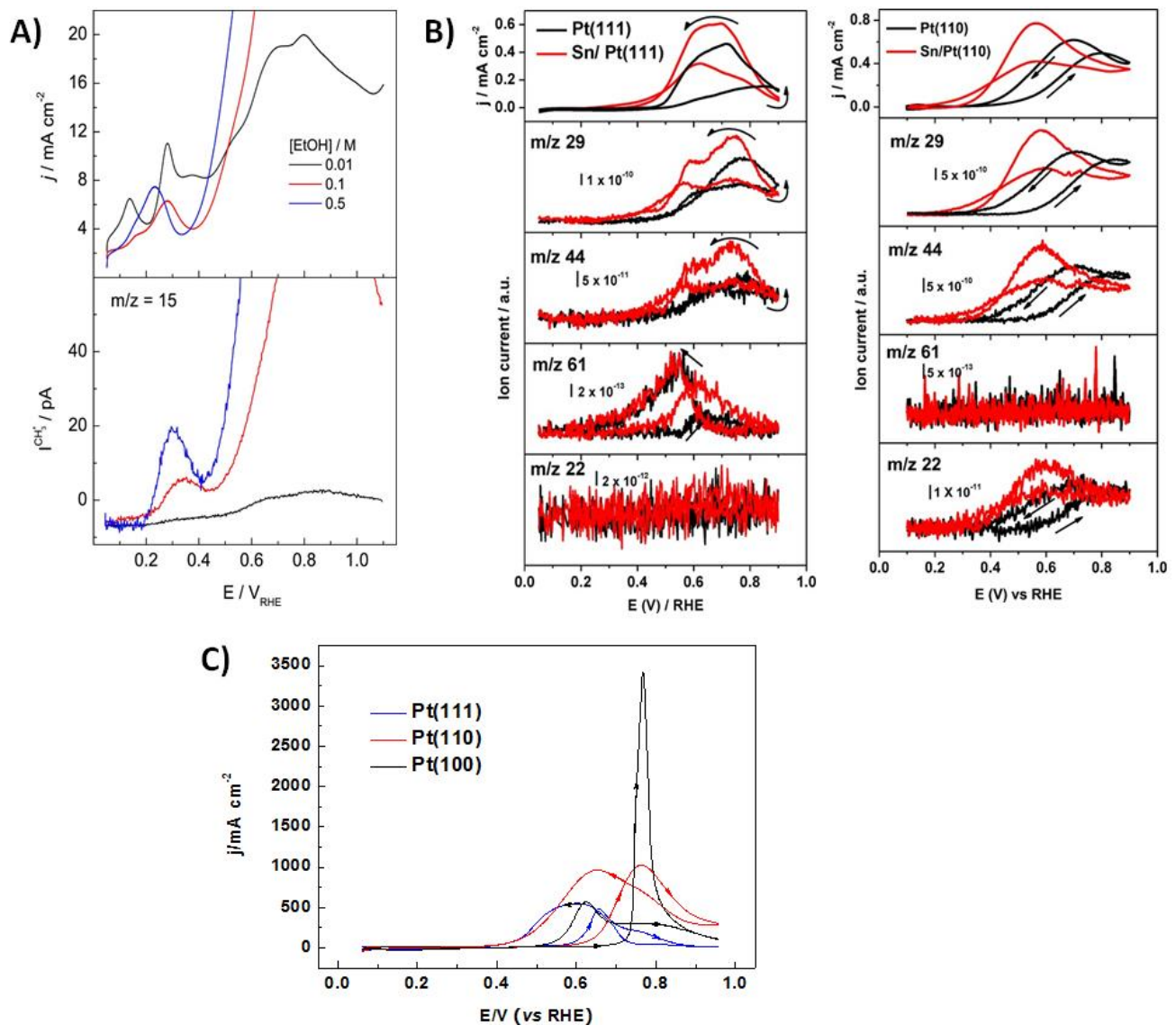

Figure 3. A) Cyclic Voltammetries (top panel) and mass spectrometer cyclic voltammetries (bottom panel, $\mathrm{m} / \mathrm{z}=15$ corresponds to $\mathrm{CH}_{3}{ }^{+}$fragment and denotes methane formation) for EOR on mesoporous Pt in $0.01,0.1$ and $0.5 \mathrm{M}$ ethanol in $0.5 \mathrm{M}$ $\mathrm{H}_{2} \mathrm{SO}_{4}$ solution. Scan rate: $2 \mathrm{mV} \mathrm{s}{ }^{-1}$. Adapted from ref [46]. B) Cyclic Voltammetries (top panels) and mass spectrometer cyclic voltammetries (bottom panels) for $\mathrm{m} / \mathrm{z}=29$ $\left(\mathrm{CHO}^{+}\right.$, acetaldehyde formation), $\mathrm{m} / \mathrm{z}=61\left(\mathrm{C}_{2} \mathrm{H}_{5} \mathrm{O}_{2}{ }^{+}\right.$, acetic acid formation) and $\mathrm{m} / \mathrm{z}=$ 
$22\left(\mathrm{CO}_{2}{ }^{++}\right.$, carbon dioxide formation) for EOR on $\mathrm{Pt}(110)$ and $\mathrm{Pt}(111)$ (black lines) and $\mathrm{Sn} / \mathrm{Pt}(110)$ and $\mathrm{Sn} / \mathrm{Pt}(111)$ (red lines) in $1 \mathrm{M} \mathrm{EtOH}+0.1 \mathrm{M} \mathrm{HClO}_{4}$; Scan rate: $1 \mathrm{mV} \mathrm{s}{ }^{-1}$. Adapted from ref. [40]. C) Stationary voltammetric profiles for the oxidation of $0.1 \mathrm{M}$ $\mathrm{EG}$ in $0.1 \mathrm{M} \mathrm{HClO}_{4}$ solution on the Pt basal planes. Scan rate: $50 \mathrm{mV} \mathrm{s}^{-1}$. Adapted from ref. [47]

Metallic adatoms have also been extensively employed to decorate the Pt single crystal surface with the aim to tune the $\mathrm{C}-\mathrm{C}$ bond cleavage and the mechanism toward EOR. Ru or $\mathrm{Sn}$ have been found to increase the oxidation currents $[40,48]$. In particular, the decoration of $\operatorname{Pt}(111)$ and $\operatorname{Pt}(110)$ with $\mathrm{Sn}$ appears to enhance the oxidation of ethanol to acetaldehyde although the further oxidation of acetaldehyde is sensitive to the Pt surface structure. On the Pt (110) surface there are sites able to break the $\mathrm{C}-\mathrm{C}$ bond and the oxidation of acetaldehyde to $\mathrm{CO}_{2}$ is promoted, whereas on $\operatorname{Pt}(111)$ such sites are not available taking place the oxidation of acetaldehyde to acetic acid (Figure 3B) [40]. Similarly, the decoration of (110) steps by $\mathrm{Sn}$ or Ru on (111) terraces also increases the activity for the oxidation behavior that is associated with an increase in the formation of acetic acid [48]. Both metals, $\mathrm{Ru}$ and $\mathrm{Sn}$ are oxophilic, and $\mathrm{OH}$ adsorption takes place at low potentials promoting the incomplete oxidation of ethanol by bifunctional mechanism with any catalytic effect of the $\mathrm{C}-\mathrm{C}$ bond splitting. On the contrary, the breaking of the $\mathrm{C}-\mathrm{C}$ bond can be favored by the modification of the Pt surface with Os [49]. FTIR experiments showed an increase of the $\mathrm{CO}$ and $\mathrm{CO}_{2}$ bands by the modification of Pt basal planes with low coverage of Os, which suggest a better capability of the $\mathrm{Pt}-\mathrm{Os}$ system to cleave the $\mathrm{C}-\mathrm{C}$ bond of ethanol, in comparison with the non-modified platinum electrodes. On the other hand, other metals such as Rh and Ir have been explored as secondary metals in Pt-based nanoparticles for EOR $[43,50]$. These studies demonstrated that the presence of both noble metals favors the cleavage 
of the $\mathrm{C}-\mathrm{C}$ when compared to the Pt pure formulation. However, as far as we know, no works on Rh and Ir modified Pt single crystals can be found in literature.

Alternatively, the $\mathrm{C}-\mathrm{C}$ bond can be destabilized when the methyl group of the EtOH molecule is partially oxidized [51-54]. In this sense, the oxidation of ethylene glycol (EG) in acid media occurs via the spontaneous and extensive $\mathrm{C}-\mathrm{C}$ bond breaking in contact with $\mathrm{Pt}$ to produce adsorbed $\mathrm{CO}$ as major surface residue $[47,51,55]$. Therefore, the introduction of a second $\mathrm{OH}$ group at the $\alpha$ carbon confers greater reactivity towards the $\mathrm{C}-\mathrm{C}$ cleavage although producing the partial blockage of the surface at lower potentials. However, the EG can also react in other reaction pathways where the $\mathrm{C}-\mathrm{C}$ bond is not altered and the $\mathrm{OH}$ groups of the molecule are sequentially oxidized. Moreover, each intermediate product can react to produce $\mathrm{C} 1$ products different from $\mathrm{CO}$, leading to an even more complex reaction mechanism that involves different reaction intermediates and products $[52,56]$. In situ spectro-electrochemical studies have provided fundamental understanding of the reaction steps during interaction of EG with $\mathrm{Pt}$, showing that adsorbed 2-hydroxyacetyl is the precursor for $\mathrm{CO}_{\mathrm{ad}}$ at low overpotentials, and that adsorbed glycolate (in equilibrium with glycolic acid under acidic conditions) results as a reaction side product/intermediate at potentials above $0.6 \mathrm{~V}$ [51,52]. On the other hand, structure-related studies have shown that within the basal planes, and similarly to EOR $[4,48]$, the $\operatorname{Pt}(111)$ electrode exhibits the best electrocatalytic properties in terms of the lowest onset potential for the oxidation of EG and little hysteresis between the positive and the negative direction scans, also pointing out the lowest poisoning rate by $\mathrm{CO}_{\mathrm{ad}}$ (Figure 3C) [47,55]. Moreover, the inclusion of steps on the (111) terrace catalyzes the EG oxidation to $\mathrm{CO}_{2}$, being the $\{110\}$ steps the most catalytic for the $\mathrm{C}-\mathrm{C}$ cleavage. Also, FTIR results showed that the incomplete oxidation towards glycolic acid and oxalic acid are produced principally at the close- 
packed terraces [51]. The selective decoration of Pt surfaces with adatoms or other adsorbed species, in combination with the higher reactivity of the $\mathrm{C}-\mathrm{C}$ bond of the $\mathrm{EG}$ molecule, could lead to a site-selective electrocatalysis and the exploit of the 10 electrons per EG molecule that can be extracted from its complete oxidation to $\mathrm{CO}_{2}$.

\section{Conclusions.}

The ideal electrocatalyst for the oxidation of these molecules should comply several properties. Platinum is one of the best electrocatalysts for the dehydrogenation, which is a required step in all the oxidation. However, the dehydrogenation requires that the molecule is adsorbed in the right configuration. For the carboxylic acid, the most stable configuration, the bidentate adsorption, is not favorable for this purpose. In the case of alcohols, the interaction of these species is very weak and only when $\mathrm{OH}$ is adsorbed on the surface, the molecule can interact with the surface and dehydrogenation can occur. For this reason, oxophylic atoms, such as $\mathrm{Ru}$ or $\mathrm{Sn}$, may help in the catalysis of these steps. These adatoms also catalyze the oxidation of $\mathrm{CO}$, which appear an intermediate after the dehydrogenation. For the cleavage of the $\mathrm{C}-\mathrm{C}$ bond, which is the most difficult step and platinum is not very active, other adatoms, such Os, Rh or Ir may be required.

\section{Acknowledgements.}

Financial support from Ministerio de Ciencia e Innovación (Project no. PID2019105653GB-I00 and FJC2018-038607-I) and Generalitat Valenciana (Projects PROMETEO/2020/063 and CDEIGENT/2019/018) is acknowledged. 


\section{Authors contribution.}

All authors have contributed equally.

\section{REFERENCES}

[1] A. Boronat-González, E. Herrero, J.M. Feliu, Heterogeneous electrocatalysis of formic acid oxidation on platinum single crystal electrodes, Curr. Opin. Electrochem. 4 (2017) 26-31.

[2] E. Herrero, J.M. Feliu, Understanding formic acid oxidation mechanism on platinum single crystal electrodes, Curr. Opin. Electrochem. 9 (2018) 145-150.

** The oxidation mechanism of formic acid is detailed for both paths.

[3] M.T.M. Koper, S.C. Lai, E. Herrero, Mechanisms of the Oxidation of Carbon Monoxide and Small Organic Molecules at Metal Electrodes, in: M.T.M. Koper (Ed.), Fuel Cell Catal. A Surf. Sci. Approach, John Wiley \& Sons, Inc., Hoboken, NJ, 2009: pp. 166-171.

[4] F. Colmati, G. Tremiliosi-Filho, E.R. Gonzalez, A. Berná, E. Herrero, J.M. Feliu, Surface structure effects on the electrochemical oxidation of ethanol on platinum single crystal electrodes., Faraday Discuss. 140 (2008) 379-97; discussion 41737.

[5] E. Pastor, T. Iwasita, D/H exchange of ethanol at platinum electrodes, Electrochim. Acta. 39 (1994) 547-551.

[6] R. Rizo, S. Pérez-Rodríguez, G. García, Well-Defined Platinum Surfaces for the Ethanol Oxidation Reaction, ChemElectroChem. 6 (2019) 4725-4738. 
** A review with the main results of ethanol oxidation on platinum single crystal electrodes.

[7] A.P.M. Camargo, B.A.F. Previdello, H. Varela, E.R. Gonzalez, Effect of temperature on the electro-oxidation of ethanol on platinum, Química Nov. . 33 (2010) 2143-2147.

[8] R. Parsons, T. Vandernoot, The oxidation of small organic molecules: A survey of recent fuel cell related research, J. Electroanal. Chem. 257 (1988) 9-45.

[9] V. Grozovski, F.J. Vidal-Iglesias, E. Herrero, J.M. Feliu, Adsorption of formate and its role as intermediate in formic acid oxidation on platinum electrodes, ChemPhysChem. 12 (2011) 1641-1644.

[10] A. Cuesta, G. Cabello, C. Gutierrez, M. Osawa, Adsorbed formate: the key intermediate in the oxidation of formic acid on platinum electrodes, Phys. Chem. Chem. Phys. 13 (2011) 20091-20095.

[11] A. Ferre-Vilaplana, J.V. V. Perales-Rondón, C. Busó-Rogero, J.M. Feliu, E. Herrero, Formic acid oxidation on platinum electrodes: a detailed mechanism supported by experiments and calculations on well-defined surfaces, J. Mater. Chem. A. 5 (2017) 21773-21784.

** DFT calcualtions on the mechanism in support to the experimental results.

[12] A. Betts, V. Briega-Martos, A. Cuesta, E. Herrero, Adsorbed Formate is the Last Common Intermediate in the Dual-Path Mechanism of the Electrooxidation of Formic Acid, ACS Catal. 10 (2020) 8120-8130.

* Experimental resutls showing that formate is the bifucation point in the oxidation 
mechanis,

[13] A. Cuesta, Formic acid oxidation on metal electrodes, Encycl. Interfacial Chem. Surf. Sci. Electrochem. 5 (2018) 620-632.

[14] A. Cuesta, Electrooxidation of C1 organic molecules on Pt electrodes, Curr. Opin. Electrochem. 4 (2017) 32-38.

* The oxidation mechamism of $\mathrm{C} 1$ moelecues $(\mathrm{CO}$, formic acid and methanol) are comapred.

[15] J. Xu, D. Yuan, F. Yang, D. Mei, Z. Zhang, Y.-X. Chen, On the mechanism of the direct pathway for formic acid oxidation at a Pt (111) electrode, Phys. Chem. Chem. Phys. 15 (2013) 4367-4376.

[16] H.-F. Wang, Z.-P. Liu, Formic Acid Oxidation at Pt/H2O Interface from Periodic DFT Calculations Integrated with a Continuum Solvation Model, J. Phys. Chem. C. 113 (2009) 17502-17508.

[17] A. Ferre-Vilaplana, J.V. Perales-Rondón, J.M. Feliu, E. Herrero, Understanding the effect of the adatoms in the formic acid oxidation mechanism on $\operatorname{Pt}(111)$ electrodes, ACS Catal. 5 (2015) 645-654.

* The effect of the adatoms in the oxidation mechanism of formic acid is untangled.

[18] J. V. Perales-Rondón, S. Brimaud, J. Solla-Gullón, E. Herrero, R. Jürgen Behm, J.M. Feliu, Further Insights into the Formic Acid Oxidation Mechanism on Platinum: pH and Anion Adsorption Effects, Electrochim. Acta. 180 (2015) 479485. 
[19] J. V. Perales-Rondón, E. Herrero, J.M. Feliu, Effects of the anion adsorption and $\mathrm{pH}$ on the formic acid oxidation reaction on Pt(111) electrodes, Electrochim. Acta. 140 (2014) 511-517.

[20] V. Briega-Martos, J. Solla-Gullón, M.T.M. Koper, E. Herrero, J.M. Feliu, Electrocatalytic enhancement of formic acid oxidation reaction by acetonitrile on well-defined platinum surfaces, Electrochim. Acta. 295 (2019) 835-845.

[21] A. Boronat-González, E. Herrero, J.M. Feliu, Fundamental aspects of HCOOH oxidation at platinum single crystal surfaces with basal orientations and modified by irreversibly adsorbed adatoms, J. Solid State Electrochem. 18 (2014) 11811193.

[22] J.V. Perales-Rondón, A. Ferre-Vilaplana, J.M. Feliu, E. Herrero, Oxidation mechanism of formic acid on the bismuth adatom-modified Pt (111) surface, J. Am. Chem. Soc. 136 (2014) 13110-13113.

[23] B. Beden, A. Bewick, C. Lamy, A study by electrochemically modulated infrared reflectance spectroscopy of the electrosorption of formic acid at a platinum electrode, J. Electroanal. Chem. Interfacial Electrochem. 148 (1983) 147-160.

[24] A. Miki, S. Ye, M. Osawa, Surface-enhanced IR absorption on platinum nanoparticles: an application to real-time monitoring of electrocatalytic reactions, Chem. Commun. (Cambridge, United Kingdom). (2002) 1500-1501.

[25] Y.X. Chen, A. Miki, S. Ye, H. Sakai, M. Osawa, Formate, an active intermediate for direct oxidation of methanol on Pt electrode, J. Am. Chem. Soc. 125 (2003) $3680-3681$. 
[26] C. Busó-Rogero, A. Ferre-Vilaplana, E. Herrero, J.M. Feliu, The role of formic acid/formate equilibria in the oxidation of formic acid on Pt (111), Electrochem. Commun. 98 (2019) 10-14.

[27] M.A. Kamyabi, R. Martínez-Hincapié, J.M. Feliu, E. Herrero, Effects of the Interfacial Structure on the Methanol Oxidation on Platinum Single Crystal Electrodes, Surfaces. 2 (2019) 177-192.

** It shows that the MOR only takes place on the OH covered surface.

[28] A.A. El Shafei, R. Hoyer, L.A. Kibler, D.M. Kolb, Methanol oxidation on Rumodified preferentially oriented Pt electrodes in acidic medium, J. Electrochem. Soc. 151 (2004) F141-F145.

[29] E. Herrero, K. Franaszczuk, A. Wieckowski, Electrochemistry of methanol at low index crystal planes of platinum. An integrated voltammetric and chronoamperometric study, J. Phys. Chem. 98 (1994) 5074-5083.

[30] T.H.M. Housmans, M.T.M. Koper, Methanol oxidation on stepped Pt [n (111)×(110)] electrodes: a chronoamperometric study, J. Phys. Chem. B. 107 (2003) 8557-8567.

[31] V. Grozovski, V. Climent, E. Herrero, J.M. Feliu, The role of the surface structure in the oxidation mechanism of methanol, J. Electroanal. Chem. 662 (2011) 43-51.

[32] V. Del Colle, P.B. Perroni, J.M. Feliu, G. Tremiliosi-Filho, H. Varela, The Role of Surface Sites on the Oscillatory Oxidation of Methanol on Stepped Pt [n (111)×(110)] Electrodes, J. Phys. Chem. C. 124 (2020) 10993-11004. 
[33] A. Cuesta, At least three contiguous atoms are necessary for $\mathrm{CO}$ formation during methanol electrooxidation on platinum, J. Am. Chem. Soc. 128 (2006) 13332-13333.

[34] M. Neurock, M. Janik, A. Wieckowski, A first principles comparison of the mechanism and site requirements for the electrocatalytic oxidation of methanol and formic acid over Pt, Faraday Discuss. 140 (2009) 363-378.

[35] D. Cao, G.-Q. Lu, A. Wieckowski, S.A. Wasileski, M. Neurock, Mechanisms of methanol decomposition on platinum: A combined experimental and ab initio approach, J. Phys. Chem. B. 109 (2005) 11622-11633.

[36] L. Ou, J. Chen, Y. Chen, J. Jin, Mechanistic Study of Pt-Catalyzed Electrooxidation of $\mathrm{HCOOH}$ in Acid Medium: Kinetic Considerations on the Effect of Solvation, J. Phys. Chem. C. 122 (2018) 24871-24884.

[37] K. Franaszczuk, E. Herrero, P. Zelenay, A. Wieckowski, J. Wang, R.I.I. Masel, A comparison of electrochemical and gas-phase decomposition of methanol on platinum surfaces, J. Phys. Chem. 96 (1992) 8509-8516.

[38] M. Watanabe, S. Motoo, Electrocatalysis by ad-atoms: Part III. Enhancement of the oxidation of carbon monoxide on platinum by ruthenium ad-atoms, J. Electroanal. Chem. 60 (1975) 275-283.

[39] M. Watanabe, S. Motoo, Electrocatalysis by ad-atoms: 2. Enhancement of oxidation of methanol on platinum by ruthenium ad-atoms, J. Electroanal. Chem. 60 (1975) 267-273.

[40] R. Rizo, M.J. Lázaro, E. Pastor, M. Koper, Ethanol Oxidation on Sn-modified Pt 
Single-Crystal Electrodes: New Mechanistic Insights from On-line

Electrochemical Mass Spectrometry, ChemElectroChem. 3 (2016) 2196-2201.

[41] A. Ferre-Vilaplana, C. Busó-Rogero, J.M. Feliu, E. Herrero, Cleavage of the C-C Bond in the Ethanol Oxidation Reaction on Platinum. Insight from Experiments and Calculations, J. Phys. Chem. C. 120 (2016) 11590-11597.

[42] C. Busó-Rogero, E. Herrero, J.M. Feliu, Ethanol Oxidation on Pt Single-Crystal Electrodes: Surface-Structure Effects in Alkaline Medium, ChemPhysChem. 15 (2014) 2019-2028.

[43] Q. Chang, S. Kattel, X. Li, Z. Liang, B.M. Tackett, S.R. Denny, P. Zhang, D. Su, J.G. Chen, Z. Chen, Enhancing C-C Bond Scission for Efficient Ethanol Oxidation using PtIr Nanocube Electrocatalysts, ACS Catal. 9 (2019) 7618-

* The effect of Ir in the C-C bond cleavage is studied.

[44] F. Colmati, G. Tremiliosi-Filho, E.R. Gonzalez, A. Berná, E. Herrero, J.M. Feliu, G. Tremiliosi, E.R. Gonzalez, A. Berna, E. Herrero, J.M. Feliu, The role of the steps in the cleavage of the $\mathrm{C}-\mathrm{C}$ bond during ethanol oxidation on platinum electrodes, Phys. Chem. Chem. Phys. 11 (2009) 9114-9123.

[45] M.J.S. Farias, W. Cheuquepán, A.A. Tanaka, J.M. Feliu, Unraveling the Nature of Active Sites in Ethanol Electro-oxidation by Site-Specific Marking of a Pt Catalyst with Isotope-Labeled 13CO, J. Phys. Chem. Lett. 9 (2018) 1206-1210.

[46] J. Flórez-Montaño, G. García, O. Guillén-Villafuerte, J.L. Rodríguez, G.A. Planes, E. Pastor, Mechanism of ethanol electrooxidation on mesoporous Pt electrode in acidic medium studied by a novel electrochemical mass spectrometry 
set-up, Electrochim. Acta. 209 (2016) 121-131.

** The formation of $\mathrm{CH}_{4}$ from ethanol at low potentials is observed.

[47] R.M. Arán-Ais, N. Abe Santos, H.M. Villulas, J.M. Feliu, Oxidation of Ethanol and Its Derivatives on Well Defined Pt Single Crystal Electrodes Vicinal to Pt(111): A Comparative Study, ECS Trans. 53 (2013) 11-22.

[48] J. Souza-Garcia, E. Herrero, J.M. Feliu, Breaking the C-C bond in the ethanol oxidation reaction on platinum electrodes: Effect of steps and ruthenium adatoms, ChemPhysChem. 11 (2010) 1391-1394.

** It shows that the celaves og the C-C bond only takes at the steps and the fragments from this cleavae evolve in different ways.

[49] V. Pacheco Santos, G. Tremiliosi-Filho, Effect of osmium coverage on platinum single crystals in the ethanol electrooxidation, J. Electroanal. Chem. 554-555 (2003) 395-405.

[50] N. Erini, S. Rudi, V. Beermann, P. Krause, R. Yang, Y. Huang, P. Strasser, Exceptional activity of a Pt-Rh-Ni ternary nanostructured catalyst for the electrochemical oxidation of ethanol, ChemElectroChem. 2 (2015) 903-908.

* Effect of Rh in the cleavage of the $\mathrm{C}-\mathrm{C}$ bond in ethanol.

[51] R.M. Arán-Ais, E. Herrero, J.M. Feliu, The breaking of the CC bond in ethylene glycol oxidation at the $\operatorname{Pt}(111)$ electrode and its vicinal surfaces, Electrochem. Commun. 45 (2014) 40-43.

* It shows that the second $\mathrm{OH}$ group in the $\mathrm{C} 2$ molecule destabilizes the $\mathrm{C}-\mathrm{C}$ bond. 
[52] J. Schnaidt, M. Heinen, Z. Jusys, R.J. Behm, Oxidation of the Partly Oxidized Ethylene Glycol Oxidation Products Glycolaldehyde, Glyoxal, Glycolic Acid, Glyoxylic Acid, and Oxalic Acid on Pt Electrodes: A Combined ATR-FTIRS and DEMS Spectroelectrochemical Study, J. Phys. Chem. C. 117 (2013) 1268912701.

[53] R.M. Arán-Ais, E. Herrero, J.M. Feliu, Thermodynamic studies of anion adsorption at the $\operatorname{Pt}(111)$ electrode surface from glycolic acid solutions, J. Solid State Electrochem. 19 (2014) 13-21.

[54] R. Martínez-Hincapié, R.M. Arán-Ais, J.M. Feliu, Weakening the C[sbnd]C bond: On the behavior of glyoxylic acid on Pt(111) and its vicinal surfaces, J. Electroanal. Chem. 779 (2016) 75-85.

[55] J.M. Orts, A. Fernandez-Vega, J.M. Feliu, A. Aldaz, J. Clavilier, Electrochemical oxidation of ethylene glycol on Pt single crystal electrodes with basal orientations in acidic medium, J. Electroanal. Chem. Interfacial Electrochem. 290 (1990) $119-133$.

[56] B. Wieland, J.P. Lancaster, C.S. Hoaglund, P. Holota, W.J. Tornquist, Electrochemical and infrared spectroscopic quantitative determination of the platinum-catalyzed ethylene glycol oxidation mechanism at $\mathrm{CO}$ adsorption potentials, Langmuir. 12 (1996) 2594-2601. 